\title{
EARLY POST-ACUTE CLINICAL and EVOLUTIVE PARTICULARITIES of CERVICAL SPINAL CORD INJURY (CSCI) at ELDERLY SUBJECTS
}

\author{
Aurelian Anghelescu, Gelu Onose, Anca Sanda Mihaescu \\ Neuromuscular Rehabilitation Clinic, Teaching Emergency Hospital "Bagdasar-Arseni" \\ Bucharest \\ aurelian anghelescu@yahoo.co.uk
}

\section{Summary}

Study design: Retrospective analysis, focused on the early post acute evolution of 291 elderly ( $>65$ years) patients with CSCI, first time admitted in the Rehabilitation Clinic, during 2004-2013.

Methods: analysis of the medical files.

Results: There were 205 males and 86 females (ratio 2,4). Most of the patients (215 - 74\%) were from rural regions, and 57\% of the CSCI happened during agricultural activity; the etiology was dominated (79 \%) by accidental falls from the same level (22\%), falls from height (from chariot 48\%, from trees 5,5\%), but also traffic accidents (21\%).

Vertebral lesions consisted in facet dislocation (bilateral in 32\%, unilateral in 11\% cases) or/and (associated) disk hernia (54\%); about $37 \%$ patients had no radiological evidence of bone injury.

Conservative management was indicated in 106 subjects (36,4\%) vs. operated (63,6\%), with significant improvement of the neurological status in the surgical approached group, versus the non-operated (Fisher test, $\mathrm{p}<0.01$ ). Early admission in our department and specialized treatment favored a better neurological outcome, from totally paralisis (AIS type $\mathrm{A}+\mathrm{B}$ ) to incomplete lesions (AIS type $\mathrm{C}+\mathrm{D})(\mathrm{p}=0.006)$.

Most of the patients $175(60 \%)$ were discharged at home, whereas $63(21,6 \%)$ were admitted in nursing home, aspect significantly correlated with the marital status vs. single (widow or divorced) $(p<0,01)$.

A low mortality rate $(2 \%)$ and miscellaneous medical complications were noticed, mainly transient postural hypotension 192 subjects (65,9\%), asymptomatic urinary tract infections 259 (89\%), haematuria (42 14,4\%), bronchopneumonia (11,3\%), depression (58,7\%), central pain (44\%), mild decubitus ulcer 7 patients $\quad(2,4 \%)$.

Conclusions: CSCI at elderly has etiological and physiopathological particularities. Geriatric associated pathology and poor social conditions favored the incidence of CSCI. Comprehensive inter- / multidisciplinary therapeutic approach, early admission in the rehabilitation department after surgical stabilization, favored a better outcome and a low mortality rate.

Key words: cervical spinal cord injury, elderly, early post acute evolution, medical complications, mortality rate, comprehensive inter- / multidisciplinary therapeutic approach 